\title{
Image Dehazing Based on Physical Model and Gray Projection
}

\author{
Joxy John, Swapna Davies
}

\begin{abstract}
The climatic scattering and ingestion offer climb to the ordinary marvel of obscurity, which truly impacts the detectable quality of view. Dehazing is the technique used to expel the dimness. In late year, various works have been done to improve the detectable quality of picture taken under horrible climate. The images that are taken under overcast conditions experience the evil impacts of shading contortion and attenuation. The proposed strategy is in light of the Dark Channel Prior speculation and gray projection. The transmission map is resolved using the determined estimation of atmospheric light. It uses box filter to lessen the complexity and to improve the computing speed. This computation can restore image with incredible quality and the speed of image computation is high. The proposed strategy is differentiated with other image enhancement strategies and image restoration techniques. It is likewise exceptionally proficient technique since it can process huge images within less time.
\end{abstract}

Keywords: Dark Projection, Image dehazing, Physical Model, Transmission estimation.

\section{INTRODUCTION}

Humans are able to see the objects as light is reflected from them. The reflected light is absorbed or scattered by the air. If the air contains additional particles like fog, mist, the visibility of the object reduces. Thus, original light is replaced by the reflected light. This results in hazed image. Dehazing is the way toward expelling cloudiness. Various methods are there to increase the image quality of the dehazed image. A fast single dehazing method is proposed in view of dark channel Prior hypothesis. In the existing methodologies, the quality of restored image is poor and needs a large computation time. Based on atmospheric scattering model, the hazy image consists of both the attenuation part and scattering part. The motivation behind image dehazing is to recover dehazed image from hazed image. According to Dark channel prior, at least one-color channel of an RGB image has some pixels of low intensities, which tend to zero. This method cannot be associated with white regions, for example, sky territories and submerged surfaces. The proposed technique is based on gray projection and physical model. The speed of computation is fast and the quality of the dehazed image will be high. This strategy is partitioned into three stages. Firstly, evaluation of the transmission map using filters. Secondly, to gauge the atmospheric light the gray projection technique is used. Thirdly, the image is restored

Revised Manuscript Received on December 16, 2019

Joxy John, Electronics and Communication Engineering, Rajagiri School of Engineering and Technology, Ernakulam, India.

Swapna Davies, Electronics and Communication Engineering, Rajagiri School of Engineering and Technology, Ernakulam, India. using the Weber Fechner Law, to modify the splendor of the image. The brightness is adjusted in order to get the restored image clearer and more natural.

\section{RELATED WORKS}

\section{A. ATMOSPHERIC SCATTERING MODEL}

In atmospheric scattering the little particles suspended in a vehicle of refractive index diffuse a segment of the incident radiations in all directions. The dissipating model to portray the hazy image can be imparted as

$$
I(x)=J(x) t(x)+A(1-t(x))
$$

Where $\mathrm{x}$ is the separation co-ordinate, $\mathrm{I}(\mathrm{x})$ is the foggy image, $\mathrm{J}(\mathrm{x})$ is the fog free image, $\mathrm{A}$ is the climatic light, and $t(x)$ is the transmission rate of the medium. The reason for image dehazing is to recoup $\mathrm{J}(\mathrm{x})$ from $\mathrm{I}(\mathrm{x})$.

\section{B. DARK CHANNEL PRIOR THEORY}

In Dark Channel Prior theory, at least one-color channel of an RGB image has some pixels of lowest intensities, which tends to zero. When the pixel intensity is low, the color diverges towards black. The RGB image splits into Red, Green and Blue channels, select the minimum intensity pixels. Prior is the way towards choosing the minimum intensity pixels from an RGB image. Deformities of DCP: The calculation is proposed by $\mathrm{He} 2$ has the accompanying restriction:

(1) It utilizes delicate tangling to refine transmission map, brings about low computational speed.

(2) The strategy cannot be associated with white zones, for example, sky zones and submerged surfaces.

\section{WEBER FECHNER LAW}

The law relates the original image in the physical upgrades and the apparent image. The image is restored using the corrected transmission map and tone is adjusted.

\section{SYSTEM MODEL}

The fast dehazing method is based on gray projection and the physical model is isolated into four stages. (A)Estimation of transmission map, (B)Calculation of Atmospheric light, (C)Calculation of Transmission Map, (D)Restoration of The Image and Tone adjustment. 


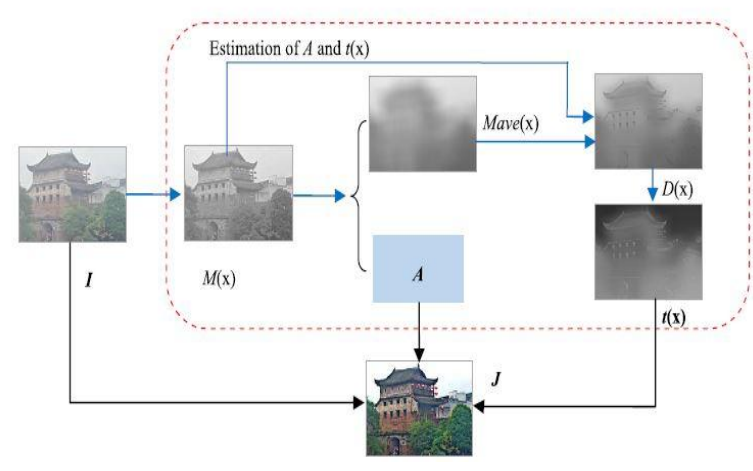

Fig. 1. Block Diagram

\section{A. ESTIMATION OF TRANSMISSION MAP}

The initial step of the calculation is to estimate the transmission map. The algorithm steps are as follows:

Step 1: The input image to the filter is the hazed image.

Step 2: To obtain the Prior Dark Channel Image minimum filter is used on the input Image. It is used to remove the positive outlier noise.

$$
M(x)=\min (I c(x))
$$

Step 3: For obtaining smoothed image $\operatorname{Mavg}(\mathrm{x})$. The obtained dark channel prior image undergoes a filter averaging.

$$
\operatorname{Mavg}(x)=\operatorname{average}(M(x))
$$

Where $\square$ is the size of filter window, which is taken as the $1 / 20$ of image width.

Step 4: To obtain the corrected image $\mathrm{D}(\mathrm{x})$, grayscale filter is operated on the smoothened image.

$$
D(x)=\min (A * \operatorname{Mavg}(x)), M(x))
$$

On passing through the average filter, there will be a gray trend in the image. To reduce the gray trend in the image grayscale optimization is done. To improve the figuring speed, the integral image is utilized. For increasing the calculational speed, an Integral Image is used. The Integral Image is used as a lively and convincing technique for processing the total of characteristics (pixel regards) in a given image. For an original image $i(x, y)$, the Integral image is denoted as ii $(x, y)$. A box filter is utilized to expand the count speed by a factor of four dependent on integral image. In contrast, the information stored in the matrix are straightforwardly the whole of current pixel and the neighboring pixels. The relating component in the framework would then be able to be legitimately accessed, which brings about zero complexity.

\section{B. CALCULATION OF ATMOSPHERIC LIGHT}

To discover pixels with high brightness and smoothness, the projection technique is utilized for area isolation. If there is a white area in the scene, if the window determination during the procurement of channel picture is not appropriate, the white area will remain and make the picked power of barometrical light be prevalently founded on that object. In open air pictures, the sky parcel for the most part has the attributes of enormous territory, splendid shading and smoothness.

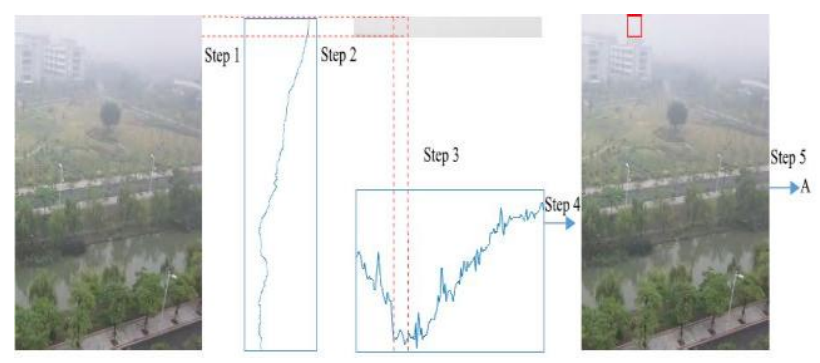

Fig. 2. Calculation of Atmospheric Light

The steps in the algorithm includes the following:

Step 1: Horizontal projection of the image.

$$
H(y)=\sum_{x=1}^{m} I(x, y) \quad 1 \leq x \leq m
$$

Step 2: Sum the projection within a width of $2 b+1$ and selects the maximum region.

$$
H \max =\max \left(\sum_{y=s-b}^{s+b} H(y) b+1 \leq s \leq m-b\right.
$$

Step 3: Vertical projection of the minimum filtered image.

$$
V(x)=\sum_{x=1}^{n} K(x, y) \quad 1 \leq y \leq 2 b+1
$$

Step 4: Sum the projection within a width of $2 b+1$ and selects the maximum region.

$$
V \max =\max \left(\sum_{x=s-b}^{s+b} V(x)\right) b+1 \leq s \leq n-b
$$

The maximum region is saved as $\mathrm{R}(\mathrm{x})$.

Step 5: Calculate atmospheric light.

$$
A=\operatorname{mean}(\max 0.1 R(x))
$$

The pixel values of the sky region are extracted first and is arranged in descending order. The average gray values of the top one percent pixels with large brightness values are selected and applied to the formula. The region after computation is saved as $\mathrm{R}(\mathrm{x})$, repeated summation over certain region can be performed such that there will be reduction in computational steps.

\section{CALCULATION OF TRANSMISSION MAP}

The transmission map is calculated, if the value of A is known. The transmission map needs to be calculated, to restore a haze free image $\mathrm{J}(\mathrm{x})$ from the hazed image $\mathrm{I}(\mathrm{x})$.

For restoring the haze-free image from hazy image the value of $t(x)$ is required. In the estimation of transmission map, the transmission map is calculated after calculating the value of atmospheric light.

The DCP superposition that is wasteful in featured area, which results in transmission rate to be little and almost equivalent to zero. To evade shading contortion the sky and non-sky zones are segmented. To get an increasingly precise transmission conveyance of splendid territory, the recipe can be expressed as 


$$
T=0.15 \quad(A-\operatorname{Im}) \leq 0.25
$$

The value of $\mathrm{T}$ is taken as 0.15 and the average gray of the image is calculated.

$$
\operatorname{Im}=\operatorname{mean}(I(x))
$$

The maximum difference between the three RGB channels and atmospheric light is calculated to get the corrected transmission map. The corrected transmission map is calculated by comparing the maximum difference and threshold value. The threshold value is set to 0.2 as it gives more accurate result for sky area. The proposed technique can accommodate hazed images with enormous splendid regions with no takeoff from the DCP suspicion. This adjustment presents constrained changes on the first calculation.

\section{RESTORATION OF IMAGE AND TONE ADJUSTMENT}

The image can be restored using the formula, when $\mathrm{t}(\mathrm{x})$ tactics zero, the restored image may contain noise after estimating the transmission map and atmospheric light $\mathrm{A}$. When $t(x)$ tactics zero, the restored photograph may additionally include noise. To keep away from haze content between the restored image a lower bound to $=0.1$ is employ in the calculation. $\mathrm{J}(\mathrm{x})$ is the last dehazed image. The Weber-Fechner Law relates to human perception. Because of atmospheric changes, there is a relation between actual brightness and perceived brightness of an image. The logarithmic linear relation of Weber-Fechner Law is expressed as

$$
J d={ }_{\beta} \lg (J)+{ }_{\beta} 0
$$

Where ${ }_{\beta}$ and ${ }_{\beta} 0$ are constants.

The law relates the original image in the physical upgrades and the apparent image. To avoid the computational complexity of logarithmic function, it adopts a simple function and adjusts the restored tone.

\section{SUBJECTIVE EVALUATION}

\section{A. COMPARISON WITH IMAGE ENHANCEMENT BASED METHODS}

The technique suggested is contrasted with various image enhancement methods.

1) Contrast stretching: - It is a simple image enhancement approach chronic in imitation of decorate photo distinction by means of extending the variety over depth values it consists of in imitation of cowl the required range on values.

2) Histogram Equalization: - It is used to enhance contrast by adjusting the image intensities.

3)Adaptive Histogram Equalization: - It works small regions of data instead of the entire image to enhance the contrast of an image.

4) Homomorphic Filtering: - It involves a non-linear mapping to another domain where linear methods are implemented and mapping back to the initial domain.

5) Wavelet Transform: - It is a transform operation used to decrease image entropy. It is reversible and does not result in any information loss.

6) Median Filtering: - It is a method of smoothing images by reducing the intensity values.

Since all other image enhancement methods improves the quality of image. The visual effect is superior in the proposed strategy.

\section{B. IMAGE QUALITY METRICS}

Image quality metrics- PSNR and MSE. (To know which enhancement method is better). PSNR (peak signal-to-noise ratio) is the peak error between the enhanced image and original image. MSE (mean squared error) is the difference between the pixels of two images.

$$
P S N R=20 \log 10(M A X f / \sqrt{M S E})
$$

PSNR of different image enhancement methods are calculated and the values show that the proposed method have high value which means less error and the image restored appears clearer and more natural.

\section{COMPARISON WITH IMAGE RESTORATION BASED METHODS}

The proposed technique is contrasted with other restoration methods.

1) He et al Method-The Minimum filter is ancient according to account the transmission map, it uses soft-matting according to refine the transmission chart then the computational expenses are excessive.

2) Meng et al Method- It is a single image dehazing regularization method, it uses guided image filtering Weiner filter.

3)Zhu Method- A earlier color wastage in imitation of beget a linear model. The common saturation before (ASP), as is an extensive statistical over high-definition out of doors images. Using this preceding joint together with the multiplied atmospheric scattering model, the atmospheric scattering coefficient is without delay estimated yet photo is restored.

4)Cai Method- An end to end Dehaze net, is used for transmission estimation and estimates a medium transmission map. The processing time for different restoration methods are compared. The proposed method processing time is calculated for different resolution and a graph is plotted. The table listed below shows the processing time of different image restoration methods.

On comparing with the values, the proposed system needs less processing time for large resolution images when compared to others.

For processing any sized images, the proposed approach takes solely much less processing time. The proposed approach utilizes a box a averaging filter, which increases the speed. To evaluate the extend of computing epoch because each technique along increasing image size, the relative time approach is taken into account.

$$
\operatorname{Tr}=t n / t 1
$$

Where, $\mathrm{t} 1$ is the processing time of smallest image and $\mathrm{n}$ is the image index. 


\section{Image Dehazing Based on Physical Model and Gray Projection}

\section{RESULT AND DISCUSSION}

This section includes the simulation results of the paper. Fig.3 shows the estimation of transmission map. The calculation of transmission map is only possible after the computation of atmospheric light. Fig.4 shows the calculation of atmospheric light. The value of A obtained is 0.7635. Fig.6 shows the image restoration and tone adjustments. Fig.7 shows the Comparison with image enhancement-based methods.

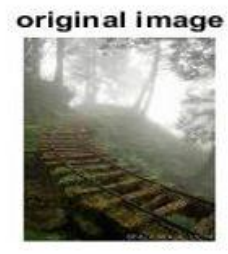

average filtering

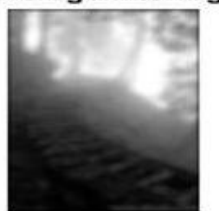

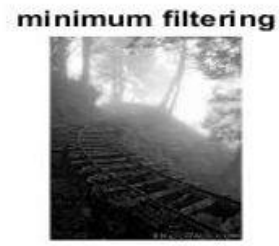

grayscale optimization

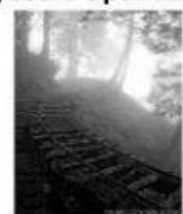

Fig. 3. Estimation of Transmission Map

horizontal projection

sum of horizontal projection

vertical projection

sum of vertical projection

Fig. 4. Calculation of atmospheric light

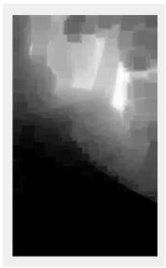

Transmission map

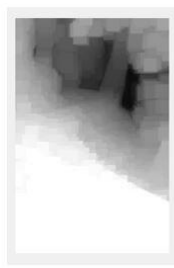

Corrected Transmission map

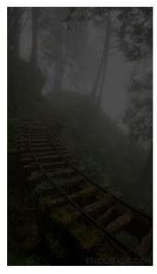

Restored image

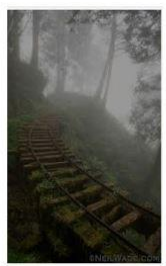

Restored image

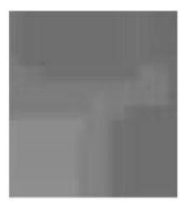

Magnification of rectangle

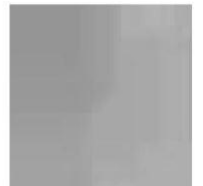

Magnification of rectangle

Fig. 5. Estimation of Transmission Map

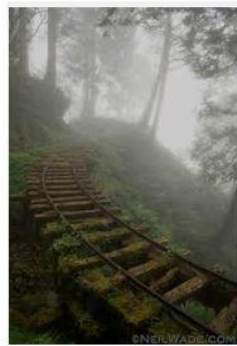

Before tone adjustment

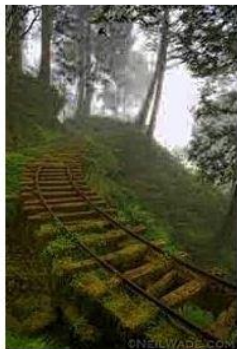

After tone adjustment

Fig. 6. Image restoration and tone adjustments

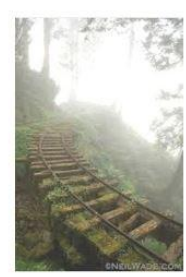

Contrast stretching

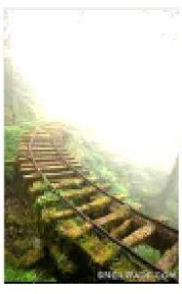

Wavelet Transform

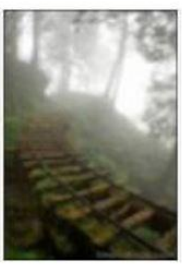

Average filtering method

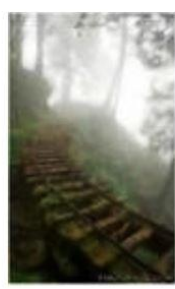

Homomorphic Filtering

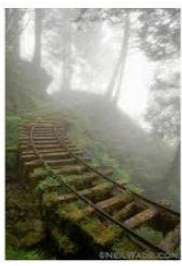

Histogram equalization

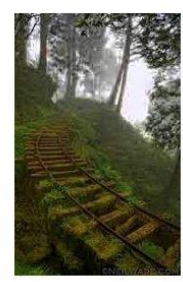

Dehazed Image
Fig. 7. Comparison with image enhancement based methods

Since all other image enhancement methods improves the quality of image. The visual effect is superior in the proposed strategy.

TABLE I

IMAGE QUALITY METRICS

\begin{tabular}{|l|c|}
\hline Type of method & $\begin{array}{c}\text { PSNR } \\
\text { Value[dB] }\end{array}$ \\
\hline Hazy 1mage & 26.6994 \\
\hline Median Filtering & 28.5740 \\
\hline Histogram & 29.4513 \\
\hline Equalization & 28.2793 \\
\hline Homomorphic & 28.6692 \\
\hline Filtering & 32.2125 \\
\hline Wavelet Transform & \\
\hline Proposed Strategy & \\
\hline
\end{tabular}

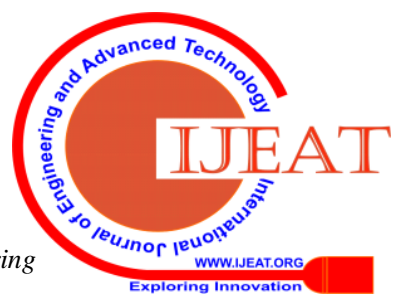


TABLE II

TIME INTERVAL FOR VARIOUS RESTORATION METHODS

\begin{tabular}{|c|c|c|c|c|c|}
\hline Resolution & $\begin{array}{c}\text { Meng } \\
\text { et.al }\end{array}$ & He.et.al & $\begin{array}{c}\text { Zhu } \\
\text { et.al }\end{array}$ & $\begin{array}{c}\text { Cai } \\
\text { et.al }\end{array}$ & Proposed \\
\hline $600 * 400$ & 1.243 & 0.584 & 0.887 & 2.130 & 0.0365 \\
\hline $800 * 600$ & 2.290 & 1.205 & 1.699 & 4.275 & 0.0403 \\
\hline $1024 * 768$ & 3.796 & 3.766 & 2.675 & 6.876 & 0.0858 \\
\hline $1600 * 1200$ & 9.380 & 7.765 & 6.546 & 17.125 & 0.1984 \\
\hline $2048 * 1536$ & 15.885 & 14.581 & 10.732 & 27.684 & 0.3246 \\
\hline
\end{tabular}

TABLE I shows the different image quality metrics. TABLE II shows the time interval for various image restoration methods.

Fig. 8 shows the time interval for various image restoration methods. With increasing image size, the relative time interval also increases. The proposed strategy requires less time for processing large images when compared with other methods. The He2 technique suggests maximum slope. Zhus method, Meng method, Cais technique hold similar rate of changes. The proposed technique can process even large images and provides excessive effectivity of implementation.

Fig. 9 shows the relative speed of variation for increasing image sizes. In all other methods, the processing time increases for increasing image resolution. The proposed strategy utilizes only less computing time even for processing large sized images. From the graph, it is clear that the complexity of the proposed strategy is less compared to other methods.

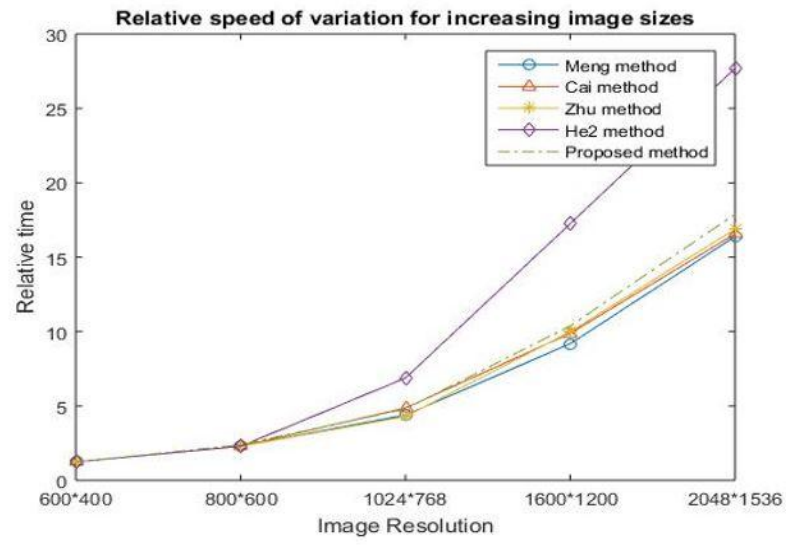

Fig. 8. Time interval for various image restoration methods

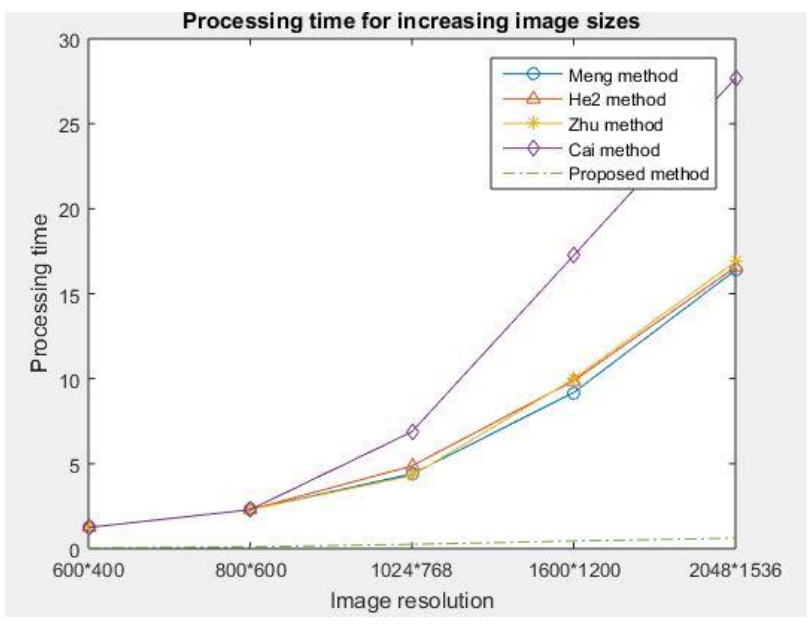

Fig. 9. Relative speed of variation for increasing image sizes

\section{CONCLUSION}

The image dehazing based on Physical Model and Gray projection improves the quality of restored image and the speed of computation when compared with other methods. It makes use of box acceleration filter such that it reduces the computational steps. It needs only less processing time for computing even large images. The proposed strategy is faster than other existing dehazing methods.

\section{REFERENCES}

1. Wencheng Wang, Tao Ji, Faliang Chang, "A Fast Single-Dehazing Method Based on Physical Model and Gray Projection”, IEEE Access, vol. 6, no. 3, Feb.2018.

2. K. He, J. Sun, X. Tang "Single image haze removal using dark channel prior", IEEE Trans. Pattern Anal. Mach. Intell, vol. 33, no. 12, Dec.2011.

3. R. Fattal, "Single image dehazing", ACM Trans. Graph, vol. 27, no. 3.p.72, Aug.2008.

4. H. Luetal, "Depth map reconstruction for underwater Kinect camera using inpainting and local image mode filtering", IEEE Access vol. 5, pp. 71157122 , Apr. 2017.

5. C. J. Q. Zhu, J. Mai, L. Shao "A fast single image haze removal algorithm using color attenuation prior", IEEE Trans. Image Process vol. 24 , no. 11 , Nov. 2015 .

6. J. Jiang, T. Hou, M. Qi "Improved algorithm on image haze removal using dark channel prior", J. Circuits Syst vol. 16, no. 2, pp.12, 2011

7. T. J. Cooper, F. A. Baqai "Analysis and extensions of the FrankleMcCann Retinex algorithm”, J. Electron. Image., vol. 13, no. 1, pp 8592, Jan.2004.

8. M.-J. Seow, V. K. Asari, "Ratio rule and homomorphic filter for enhancement of digital color image", Neurocomputing, vol. 69, nos. 79, pp.954958, Mar. 2006

9. J. P. Oakley, B. L. Satherley "Image quality in poor visibility conditions using a physical model for contrast degradation", IEEE Trans. Image Process, vol. 7, no. 2, pp. 167179, Feb. 1998.

10. S. G. Narasimhan, S. K. Nayar, "Interactive (de) weathering of an image using physical models", IEEEWorkshop Color Photometric Methods Comput. Vis., Paris, France, Oct. 2003, pp. 18.

11. S. K. Nayar, S. G. Narasimhan, "Vision in bad weather", IEEE Int Conf. Comput. Vis., Kerkyra, Greece, Sep. 1999, pp. 820827.

12. Y. Y. Schechner, S. G. Narasimhan, S. K. Nayar, "Instant dehazing of images using polarization", IEEE Conf. Comput. Vis. Pattern Recognit., Kauai, HI, USA, Dec. 2001, pp. 325332.

13. G. Meng, Y. Wang, J. Duan, S. Xiang, C. Pan, "Efficient image dehazing with boundary constraint and contextual regularization", IEEE Int. Conf. Comput. Vis., Sydney, NSW, Australia, Dec. 2013,pp. 617624.

14. S.-C. Huang, B.-H. Chen, W.-J.Wang, 'Restoration of single hazy images captured in real-world weather conditions", IEEE Trans.Circuits Syst. Video Technol, vol. 24, no. 10, pp. 18141824, Oct. 2014.

15. H. Yuan, C. Liu, Z. Guo, and Z. Sun, "A region-wised medium transmission based image dehazing method", IEEE Access, vol. 5, pp. 17351742 , Jan. 2017.

\section{AUTHORS PROFILE}

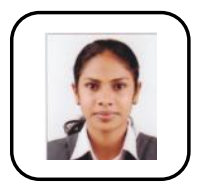

Joxy John, is a student at the department of Electronics and Communication Engineering at Rajagiri School of Engineering and Technology, Ernakulam, Kerala.

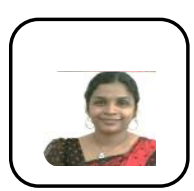

Swapna Davies, is a professor at the department of Electronics and Communication Engineering at Rajagiri School of Engineering and Technology, Ernakulam, Kerala. 